\title{
Supersonic Nanoparticle Interaction with Suspended CVD Graphene
}

\author{
J.L. Swett ${ }^{1}$, D.A. Cullen ${ }^{2}$, M. McCully ${ }^{3}$, J. A. Schultz ${ }^{3}$, P. V. Bedworth ${ }^{1}$, S. E. Heise ${ }^{1}$, S. W. Sinton ${ }^{1}$, B.

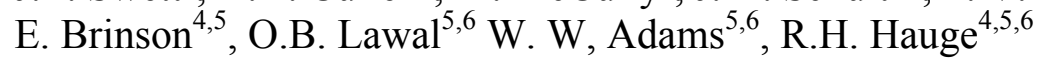 \\ ${ }^{1 .}$ Lockheed Martin Space Systems Co., Adv. Technology Center, Palo Alto, CA, USA \\ 2. Materials Science \& Technology Division, ORNL, Oak Ridge, TN, USA \\ 3. Ionwerks, Inc., Houston, TX, USA \\ 4. Rice University, Chemistry Dept., Houston, TX, USA \\ 5. Rice University, nanoCarbon Center, Houston, TX, USA \\ 6. Rice University, Material Science and Nano Engineering Dept., Houston, TX, USA
}

Supersonic nanoparticle interactions with suspended chemical vapor deposition (CVD) graphene have been probed utilizing a custom nanoparticle generation and acceleration system. By varying nanoparticle size and energy, the mechanical properties of suspended graphene under bombardment were explored at energies where nanoparticle deformation [1], fracture toughness [2], and elastic modulus [3], among other properties, result in varying interactions. These experiments help to better elucidate the potential role of graphene in ballistics applications [4] as well as the utilization of nanoparticle bombardment to modify graphene membranes for a variety of applications such as filtration and separation at the nanoscale with high permeances [5].

In this study, we utilize a custom nanoparticle generation system, an Ionwerks NPlanter, to bombard suspended graphene produced by CVD with silver nanoparticles at supersonic velocities and, in some instances, at hypervelocitiy. The instrument has the capability to size select nanoparticles at specified diameters $<30 \mathrm{~nm}$ and accelerate them at suspended graphene with energies up to $30 \mathrm{keV}$ with controlled fluence. The results from the bombardment are explored with helium ion microscopy (HIM) using a Zeiss Orion NanoFab. Detailed information on the interaction is explored further with lowvoltage, aberration-corrected scanning transmission electron microscopy (STEM) in a Nion UltraSTEM 100 utilizing both medium angle and high angle annular dark field imaging (MAADF and HAADF, respectively) to characterize the nanoparticles and graphene at atomic resolution.

We will report on novel nanoparticle interactions that we have observed, including recoil, pinning, perforation, skidding, and deformation. Additionally, novel perforations have been observed at specific energy ranges, resulting from graphene's unique mechanical properties, which exhibit different geometries and edge structures [6].

\section{References:}

[1] Kichul Yoon, Alireza Ostadhossein, Adri CT van Duin, Carbon 99 (2016), p. 58.

[2] Zhang, Peng, et al, Nature communications 5 (2014).

[3] Lee, Changgu, et al, Science 321.5887 (2008), p. 385.

[4] Wetzel, Eric D., Radhakrishnan Balu, and Todd D. Beaudet. Journal of the Mechanics and Physics of Solids 82 (2015), p. 23.

[5] Celebi, Kemal, et al, Science 344.6181 (2014), p. 289.

[6] Research supported by Independent Research \& Development funding at Lockheed Martin, Rice University's Shared Equipment Authority and the Lockheed Martin - Rice University C-LANCE 
collaborative, and through a user project supported by ORNL's Center for Nanophase Materials Sciences (CNMS), which is a Dept. of Energy, Office of Science User Facility.
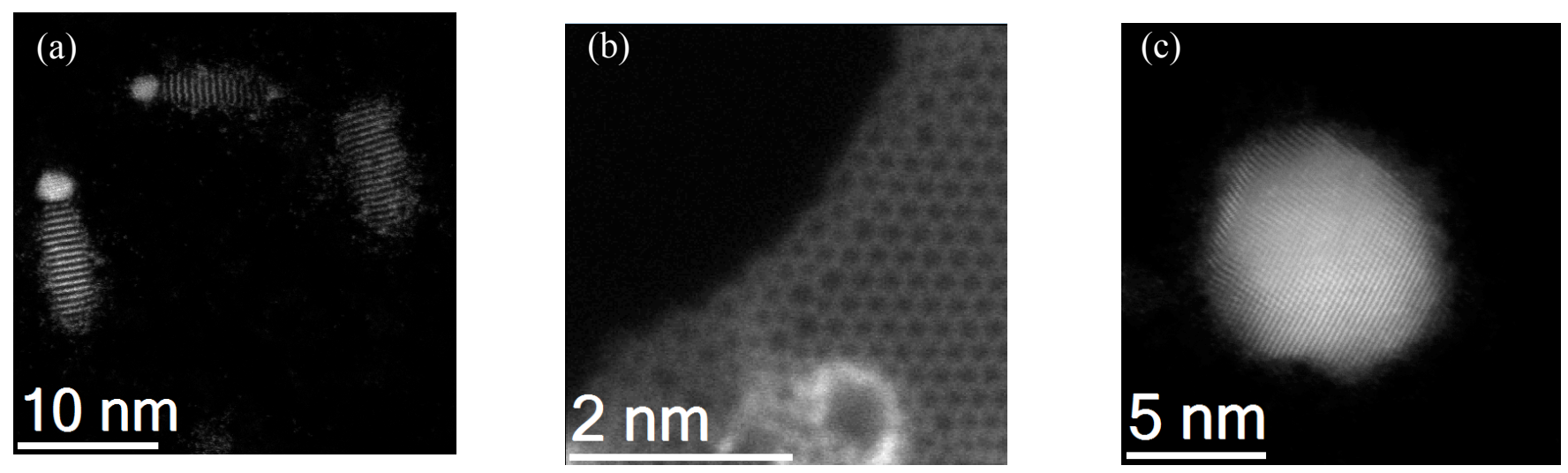

Figure 1. (a) HAADF micrograph of Ag nanoparticle "skids" on graphene. (b) MAADF micrograph of edge of a pore formed by nanoparticle bombardment. (c) HAADF micrograph of a Ag nanoparticle on graphene.
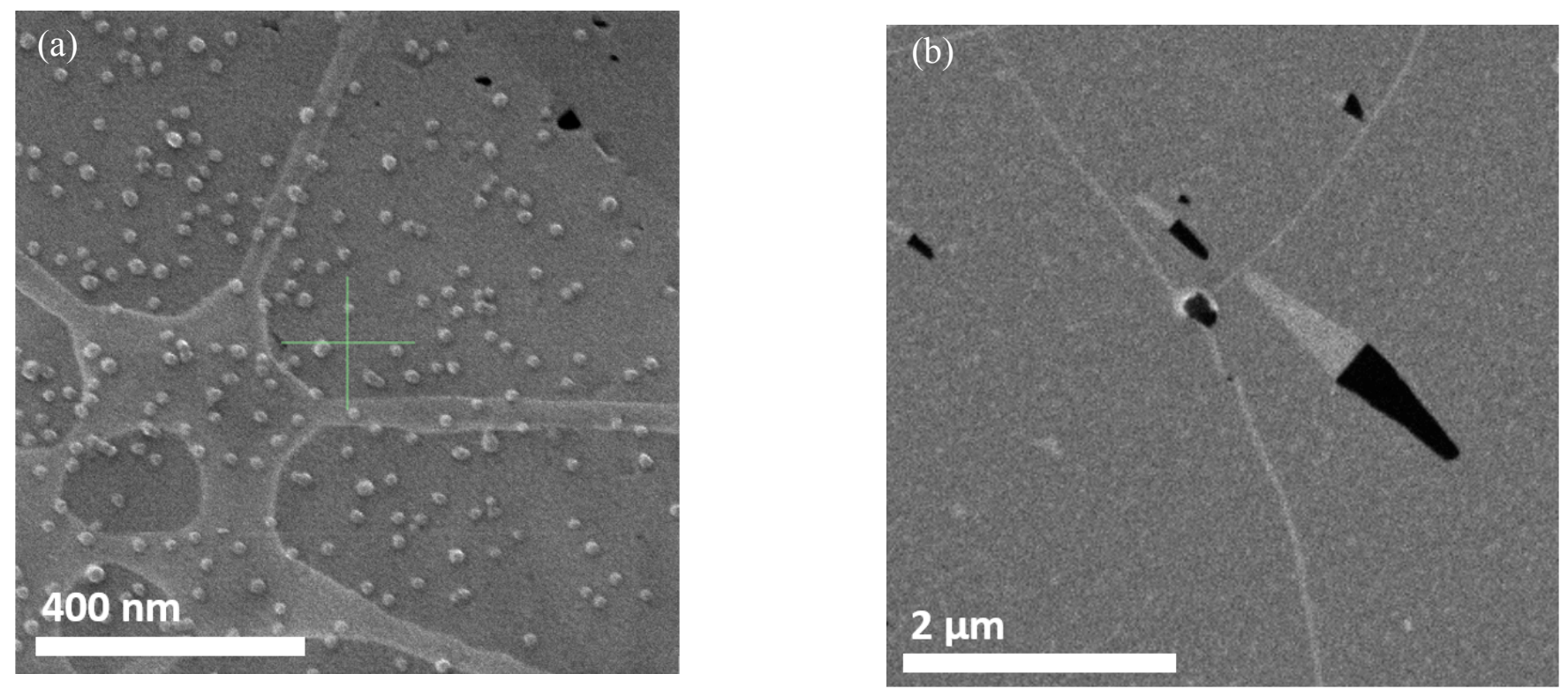

Figure 2. (a) Helium ion microscope micrograph of Ag nanoparticles on graphene. (b) Helium ion microscope micrograph showing defects resulting from silver nanoparticle bombardment of suspended graphene. 